satzleistungen auf das Blindengeld angerechnet werden. Deshalb können die staatlichen Stellen, die nach den Landes-Blindengesetzen mit einer entsprechenden Anrechnungsregelung Blindenhilfe gewähren, keinen $\mathrm{Re}-$ gress beim Schädiger aus übergegangenem Recht gemäß \116 Abs. 1 S. 1 SGB X nehmen (vgl. Plagemann, in: von Maydell/Ruland/Becker, Sozialrechtshandbuch, 5. Aufl. 2012, \$9, Rdnr. 20).

https://doi.org/10.1007/s00350-017-4763-1

\section{Anmerkung zu BGH, Urt. v. 11.4.2017 - VI ZR 454/16 (OLG Hamm)}

\section{Max Middendorf}

Das OLG Hamm (Urt. v. 9. 9.2016, - 26 U 14/16 -, Volltext abrufbar unter www.nrwe.de) hatte als Vorinstanz die Revision sowohl gemäß \543 Abs. 2 Nr. 1 ZPO (grundsätzliche Bedeutung) als auch nach Nr. 2 (Fortbildung des Rechts bzw. Sicherung einer einheitlichen Rechtsprechung) zugelassen. Dies mag auf den ersten Blick überraschen, da über die Auswirkungen von $\$ 3$ Abs. 1 GHBG NRW (nordrhein-westfälisches Gesetz über die Hilfen für Blinde und Gehörlose), also eine landesrechtliche Regelung, auf $\$ 116$ Abs. 1 S. 1 SGB X gestritten wurde. Vergleichbare Vorschriften, die die vom BGH im Leitsatz beschriebene Anrechnungsmöglichkeit enthalten, finden sich jedoch auch in anderen Bundesländern (vgl. etwa Art. 4 Abs. 3 BlindG Bayern, \$ 4 Blindengeldgesetz SchleswigHolstein, $₫ 3$ BlindGeldG Niedersachsen). Die angesprochene Problematik ist daher im Ergebnis von bundesweiter Bedeutung und erforderte eine einheitliche Vorgabe durch den BGH.

Der BGH hat mit wenigen Worten die ihm vorgelegte Fragestellung im Ergebnis zutreffend gelöst. Da die Entscheidung selbst nur sehr knapp begründet wurde, ist ein Blick auf die Erwägungen der Vorinstanz hilfreich. Das OLG Hamm hatte - anders als noch das LG Bochum - das Vorliegen der sachlichen Kongruenz im Sinne von $\$ 116$ Abs. 1 S. 1 SGB $\mathrm{X}$ als notwendige Voraussetzung für die Übergangsfähigkeit des angemeldeten Ersatzanspruches verneint. Vom Beklagten war in diesem Verfahren eingewandt worden, dass das Blindengeld im Gegensatz zum Schadensersatz für Mehraufwendungen eine bedürfnisunabhängige Pauschale darstellt. Dieser Argumentation hatte sich das OLG Hamm angeschlossen. In der Literatur und der Rechtsprechung hatte Uneinigkeit bestanden, ob eine sachliche Kongruenz zwischen dem Schadensersatz für Mehraufwendungen und dem Blindengeld gegeben ist

Zum Teil wurde in der Literatur eine sachliche Kongruenz zwischen Schadensersatz für Mehraufwendungen und Blindengeld weithin bejaht (Kater, in: Kasseler Kommentar zum Sozialversicherungsrecht, SGB X, \$116, Rdnrn. 62, 64; Bieresborn, in: von Wulffen/Schütze, SGB X, 8. Aufl., \$116, Rdnr. 5b). Dieser Einschätzung hat die Rechtsprechung sich zum Teil angeschlossen (LG Köln, VersR 2003, 751, 752; OLG München, Urt. v. 27.10.2011 - 1 U 1946/05 -, juris, Rdnr. 172). Zum Teil bestand auch eine vermittelnde Auffassung, die die Kongruenz auf diejenigen Mehraufwendungen beschränkte, die konkret dargelegt und beziffert wurden (LG Münster, Urt. v. 27. 5.2002 $15 \mathrm{O}$ 48/02 -; s. weitere Nachweise bei OLG Hamm, a.a.O., Rdnrn. 30 ff.). Entscheidend hatte die Vorinstanz

Rechtsanwalt Dr. iur. Max Middendorf,

Fachanwalt für Medizinrecht,

Bergmann und Partner mbB,

Josef-Schlichter-Allee 38, 59063 Hamm, Deutschland darauf abgestellt, dass das Blindengeld unabhängig von jeglichen Einkommens- und Vermögensverhältnissen gezahlt werde. Die völlig abstrakte Berechnung des Blindengeldes nehme für sich gar nicht in Anspruch, jeglichen Mehraufwand abzudecken.

Das OLG Hamm hatte sich insoweit auch auf das Urteil des BGH v. 30.6.2015, - VI ZR 379/14 - (abrufbar unter www.bundesgerichtshof.de) gestützt. In dem Fall ging es um die Frage, ob der Geschädigte einen Verdienstausfallschaden geltend machen konnte, obwohl seitens der Bundesagentur für Arbeit wegen der Beschäftigung des Geschädigten in einer Behindertenwerkstatt Leistungen erbracht worden waren. Der 6. Senat hatte seinerzeit dargelegt, dass auch in Fällen einer sachlichen Kongruenz zu prüfen ist, ob der Sinn und Zweck des \$116 SGB X es rechtfertigen, dass der Ersatzanspruch durch den Leistungsträger anstelle des Geschädigten geltend gemacht wird BGH, a.a.O. Rdnr. 17). Auch diesen Umstand hatte die Vorinstanz berücksichtigt. Es wurde argumentiert, dass der Geschädigte schlechter gestellt werden könnte, wenn es zu einem Anspruchsübergang käme. Eine doppelte Entschädigung des Blinden werde durch die Anrechnungsregelung des nordrheinwestfälischen Blindengeldgesetztes vermieden, wonach sich der Blinde Entschädigungsleistungen wegen Mehraufwendungen anrechnen lassen müsse.

Der BGH hat in seiner Entscheidung entscheidend darauf abgestellt, dass nach dem nordrhein-westfälischen $\mathrm{Ge}-$ setz über die Hilfen für Blinde und Gehörlose ( $\$ 3$ Abs. 1 GHBG NRW) eine Anrechnung von Leistungen stattfindet, die der Blinde nach anderen Rechtsvorschriften, und zwar auch auf bürgerlich-rechtlichen Rechtsvorschriften beruhenden Schadensersatzleistungen, erhält. Der klagende Landschaftsverband hatte diese Anrechnung auch vorgenommen. Aufgrund der Anrechnungsmöglichkeit, so der 6. Senat, könnten die Aufwendungen für die Solidargemeinschaft gering gehalten werden. Vor diesem Hintergrund sei kein Anspruchsübergang mehr erforderlich und möglich.

Die Entscheidung hat weitreichende Auswirkungen auf die Praxis. Sie ist auf alle Haftpflicht-Auseinandersetzungen anwendbar, bei denen das Opfer erblindet ist. Die Anrechnungsmöglichkeit, auf die der BGH entscheidend abgestellt hat, ist nicht nur in der nordrhein-westfälischen Regelung vorgesehen, sondern - wie oben dargelegt - auch in anderen landesrechtlichen Regelungen. Insofern ist die bundesweit bedeutsame Streitfrage, ob Sozialversicherungsträger derartige Aufwendungen regressieren können, nunmehr geklärt.

\section{Haftung für Fehler des Durchgangsarztes I}

GG Art. 34; BGB §839; SGB VII §34 Abs. 1, 3

1. Wegen des regelmäßig gegebenen inneren Zusammenhangs der Diagnosestellung und der sie vorbereitenden Maßnahmen mit der Entscheidung über die richtige Heilbehandlung sind jene Maßnahmen ebenfalls der öffentlich-rechtlichen Aufgabe des Durchgangsarztes zuzuordnen mit der Folge, dass die Unfallversicherungsträger für etwaige Fehler in diesem Bereich haften (Aufgabe der Rechtsprechung zur ,doppelten Zielrichtung“6, vgl. BGHZ 179, 115, Rdnr. 23; BGHZ $63,265,273 \mathrm{f}$.).

Eingesandt vom BGH-Entscheidungsversand, Karlsruhe bearbeitet von Rechtsanwalt Dr. iur. Patrick M. Lissel, LL.M., Fachanwalt für Medizinrecht,

Weißenseestraße 92, 81539 München, Deutschland 